\title{
TOLERANCIA Y COMPLICACIONES DE LAS BIOPSIAS PROSTATICAS ECODIRIGIDAS MEDIANTE EL USO DE UN GEL INTRARECTAL CON LIDOCAINA.
}

\author{
Miguel Álvarez-Múgica, Roberto C. González Álvarez, Antonio Jalón Monzón, Jesús M. \\ Fernández Gómez, Oscar Rodríguez Faba', Laura Rodríguez Robles y Francisco J. Regadera \\ Sejas.
}

Servicio de Urología I. Hospital Universitario Central de Asturias. Oviedo. Asturias y Servicio de Urología'. Fundación Puigvert. Barcelona. España.

\begin{abstract}
Resumen.- OBJETIVO: El objetivo de nuestro estudio, fue valorar la tolerancia por parte del paciente a una biopsia de próstata transrectal ecodirigida usando como método anestésico un gel con 2 gramos de lidocaina intrarectal, así como valorar las complicaciones de esta prueba.
\end{abstract}

MÉTODO: Durante 4 meses consecutivos se realizaron 148 biopsias de próstata ecodirigidas usando 2 gramos de lidocaina intrarectal. Se usó el mismo transductor ecográfico transrectal en todos los pacientes, usándose también el mismo mecanismo de aguja para la obtención de los cilindros. Las biopsias fueron realizadas por 7 urólogos diferentes obteniéndose en cada biopsia entre 6 y 12 cilindros. A todos los pacientes se les entregó tras la prueba un cuestionario para valorar la tolerancia a este método. Así mismo, el médico que realizó las biopsias rellenaba un cuestionario a cerca del paciente en el que valoraba la tolerancia del paciente y las complicaciones tras la prueba.

RESULTADOS: Se recogieron los datos de tolerancia en 147 casos, existiendo dolor intenso o insoportable globalmente en 25 casos (16,9\%) y nada molesto en 45 pacientes $(30,4 \%)$. Se evidenció una asociación significativa entre el resultado del cuestionario del paciente y la percepción del médico que realizó la prueba. Sólo en 10 casos el tacto rectal fue doloroso, en 13 el paso del transductor y en 15 los movimientos del mismo en el recto. Prácticamente todos los cilindros que resultaron dolorosos o insoportables en la toma de muestras fueron los recogidos de la zona del ápex. Se encontró una asociación significativa $(p=0,005)$ entre el número de cilindros y el dolor durante la obtención de los mismos, siendo mayor de lo esperado el dolor cuando se realizaron biopsias por encima de las sextantes. Sólo 14 pacientes no realizarían nuevamente la misma prueba o requerirían otro tipo de anestesia y el 89,9\% (133), volverían a realizarla en las mismas condiciones.

CONCLUSIONES: En nuestra experiencia la biopsia prostática ecodirigida es generalmente bien tolerada empleando únicamente un gel anestésico intrarectal. No obstante, el número de punciones durante la biopsia ha sido el factor asociado al dolor de la prueba y al incrementarse aquel debería plantearse el empleo de algún tipo adicional de anestesia.

Palabras clave: Adenocarcinoma de próstata. Biopsias prostáticas ecodirigidas. PSA. 
Summary.- OBJECTIVES: The objective of our study was to evaluate patient tolerance to transrectal ultrasound guided prostate biopsy using anesthesia with 2 grams of intrarectal lidocaine gel, and to evaluate the complications of the test.

METHODS: 148 prostate biopsies with intrarectal lidocaine were performed over a four month period. The same intrarectal ultrasound transducer and needle mechanism were employed for all patients. Biopsies were performed by 7 different urologists with 6 to 12 cores per biopsy. All patients received after the biopsy a questionnaire to evaluate their tolerance to the intervention. In the same way, the urologist performing the biopsy filled a questionnaire about patient tolerance and complications of the test.

RESULTS: Patient tolerance data were recorded in 147 biopsies. Twenty-five cases (16.9\%) referred severe or unbearable pain, 45 patients (13.4\%) referred no pain at all. A significant association between patients 'and doctors' results was obtained. Digital rectal examination was painful in 10 cases only; transducer insertion was painful in 13, and 15 referred pain with the transducer movements inside the rectum. Almost all painful or unbearable core biopsies were taken in the apex. There was a significant association ( $p=0.005$ ) between the number of cores per biopsy and pain, being the pain more than expected when the number of cores was greater than six. Only 14 patients would not ever repeat the same biopsy or would request a different type of anesthesia and 133 (59.9\%) of them would repeat it in the same way.

CONCLUSIONS: In our experience, transrectal ultrasound guided prostatic biopsy is generally well tolerated with intrarectal gel as the only anesthesia. Nevertheless, the number of cores taken per biopsy has been the factor associated with pain, and if the number of biopsy cores increases additional anesthesia should be considered.

Keywords: Prostate adenocarcinoma. Ultrasound guided prostate biopsy. PSA.

\section{INTRODUCCIÓN}

El cáncer de próstata es el tumor urológico más frecuente y la tercera neoplasia en el varón tras el cáncer de piel y de pulmón. La biopsia transrectal se ha convertido en la técnica estándar para su diagnóstico. Una gran mayoría de urólogos, consideran que las molestias ocasionadas por esta técnica, es muy pequeña o insignificante por lo que podría realizarse sin anestesia (1). Durante muchos años, la técnica de toma sextantes de cilindros prostáticos introducida por Hodge en 1989 fue la de elección, pero durante la última década, se han realizado numerosas modificaciones en el número de cilindros a tomar, ya que se ha demostrado recientemente que la técnica habitual de la biopsia sextante de próstata no consigue unas muestras adecuadas para la detección del cáncer de próstata (2). Por ello, se ha comenzado a realizar múltiples técnicas de biopsia, obteniendo un mayor número de cilindros prostáticos con objeto de conseguir unas mejores tasas de detección del cáncer de próstata, con una menor necesidad de biopsias repetidas (3). Recientemente, Diavan y Remzi (4) han validado el uso de un nomograma, denominado Nomograma de Viena (Tabla I), para decidir el número de cilindros prostáticos a tomar en la primera biopsia, en función de la edad del paciente y del volumen prostático cuando el valor de PSA está entre 2 y $10 \mathrm{ng} / \mathrm{ml}$. Utilizando este nomograma, se podrían tomar hasta 18 cilindros prostáticos en la primera biopsia. Esto es importante ya que el dolor aumenta a medida que aumentan el número de cilindros.

Las personas que se someten a esta prueba presentan un importante estrés psicológico por varios motivos, ya que por una parte existe el miedo al diagnóstico potencial de un cáncer, también a la vía de abordaje, y el hecho de que el órgano a examinar forme parte del aparato sexual del varón. Aunque la morbilidad asociada a este procedimiento puede suponer complicaciones menores, los pacientes perciben la prueba como un hecho traumático e invasivo (5). Se ha visto también como los pacientes con un nivel de ansiedad preprueba mayor, suelen experimentar más dolor durante la prueba (6).

Los principales factores responsables del dolor durante esta prueba son: el disconfort a nivel

TABLA I. NOMOGRAMA DE VIENA.

\begin{tabular}{|l|c|c|c|c|}
\hline $\begin{array}{c}\text { Edad (años) } \\
\text { Tamaño (cc) }\end{array}$ & $<50$ & $50-60$ & $60-70$ & $>70$ \\
\hline $20-30$ & 8 & 8 & 8 & 6 \\
\hline $30-40$ & 12 & 10 & 8 & 6 \\
\hline $40-50$ & 14 & 12 & 10 & 8 \\
\hline $50-60$ & 16 & 14 & 12 & 10 \\
\hline $60-70$ & - & 16 & 14 & 12 \\
\hline$>70$ & - & 18 & 16 & 14 \\
\hline
\end{tabular}


del ano como consecuencia de la introducción de la sonda de ultrasonidos y por los pinchazos sobre la propia glándula prostática (7), ya que estos pinchazos estimulan los receptores sensitivos de los nervios periprostáticos.

En nuestro estudio hemos querido evaluar el nivel de dolor que los pacientes experimentaban en esta prueba mediante el uso de un cuestionario realizado por nosotros, comparando éste entre pacientes que eran sometidos a una toma de biopsias sextantes, frente a otro grupo sometido a la toma de un mínimo de 8 cilindros.

\section{MATERIAL Y MÉTODOS}

Se llevó a cabo el estudio en 148 pacientes sometidos de forma consecutiva a la toma de biopsias prostática ecodirigidas durante 4 meses en el año 2005. Para la prueba se utilizó en todos los casos:

- 12,5 gramos de un gel lubricante intrarectal que contenía 2 gramos de hidrocloruro de lidocaina.
- Sonda transrectal del ecógrafo Siemens Sonoline SI250.

- Pistola para la obtención de los cilindros, marca VITESSE de la casa Optimed.

- Aguja para toma de biopsias, marca VITESSE de la casa Optimed, con corte delta, 18 G y $280 \mathrm{~mm}$ de longitud.

Las biopsias fueron realizadas por 7 médicos diferentes, si bien mas del $80 \%$ fueron hechas por 4 médicos, recogiéndose esta variable de forma codificada. Se recogió mediante un cuestionario que se suministraba al paciente tras la realización de la prueba el dolor referido por los pacientes en diferentes momentos de la biopsia. Asimismo, se recogieron en un cuestionario similar las impresiones del médico que realizaba la prueba para su comparación.

Los cuestionarios contenían las siguientes preguntas (Cuestionarios 1 y 2 ).

Los resultados fueron almacenados en una base de datos y procesados mediante el programa SPSS versión 11 de aplicaciones estadísticas.

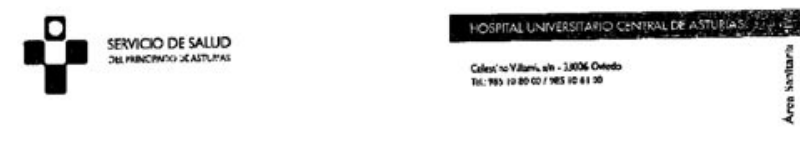

SERVICIO DJ: LROIOGIAI IIOSPITAL,CFNTRAL DE ASTURIAS

1. Antes de realizar la prucha pensaha que seria: Anies ce realizar la prucha pensaha que seria:
NADA MOLESTA / MOLIESTA / DOLOROSA / INSOPORTABI.

2. La información que ha recibido le parcece: POCA/SUFTCIRATIE/IEXTINSS

3. El grado de nerviusismo que tenia antes de la prueba era: NINGIXO / ALGO NI:RVIOSO / MIJY NTRVIOSO

4. Hil anbiente (hubitación. camilla, etc) le parves: COMOIOO/ NORMAL/ MLY INCOMOIOO

5. Fil tacto rectal $\mathrm{k}$ ha parecido: MIDA MOLESTO/MOI.ISTO/1DOL.OROSO / NSOPORTABI.I:

6. Il paso del transductor le han resultado: NADA KOL.ESTO / MOLLSTO / DOI.OROSO / NSOPORTABLE

7. Les movimientos dal transductor le han resultado: MADA MOLESTO / MOIASTO/DOL.OROSO / INSOPORTABLE

8. Lus "pincharos" de cada una de las muestras le han parecido: NADA MOLESTO / MOLISTO/I)OL.OROSO /INSOPORTABLF.

9. ¿Le han parecido todos los "pinchazos" iguales? SI NO

10. Si no le han parecido igual, $i$ a partir de cual le resultó menos tolerable:

11. Gluhalnene considera la pracba cone: NADA MOL.ESTO/MOLLSIO/DOLOROSO/INSOPORTABI.E:

12. Si ha realizade antes otras biopsins. csta le ha resultado: MÁS WOL.KSTA / IGUNL/ MENOS MOLLSTA

13. Si más adelante tuviera que hacer otra hicpsia: I.A HARIA IGUAL/ME NEG GARIA A RFPPETR / NECCESTTARIA MAS ANIESTESIA

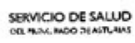

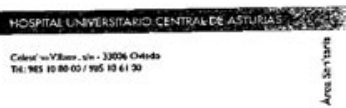

SIRVICIO DE UROHOGIA 1 HOSPTTAI. CINTRAL DE, ASILRIAS

ANITCCIDLENTES:

1. Toma de antiagragantes: SI NO

2. Toma de anticoagulantes: $\mathrm{s}$ -

4. Nivel socioculural (ESIUI)IOS): BASICOS MEDIOS SUPERIORIS

FPLORACION IIISICA

. liemerrovides: SI VO INTLRNAS IXTILRNAS AMBAS

2. lisure anal: SI VO

3. Tono del esfinter: ALTO BAJO NORMAL

4. Cirugia anal previa: SI NO

S. Otros:

TOLERANCIA A I.A PRUEBA

1. Ansiodal pre-prueba: L.EVI: MOI)LRADA IMPORTANTT

NADA YOLFSTO/MOLFSTO/DOLOROSO/NSOPORTAHI

lerancia al paso del trunsductor:

VAIM MOLIESTO/MOI.ISTO/DOL.OROSO/INSOPORTABLE

crancia a kos "pinchacos" de la biopsia:

NAIDA MOI.ESTO/MOL.\&STO/DOL.OROSO/INSOPORTABI-

Hubo dilerencias de toleruncia cnire los lóbulos prostáticos?

AMBOS IGUAL. / PEOR HI, PRIMIRO / PEOR EI. SEGIJNIOO

2. ¿Hubu al guno especial imente doloroso?

NADA MOL.ISSTO / MOI.JiSTO / DOI.OROSO / NSOPORTABL.

PROCFIIMUINTO:

. Tamaño prustático: ec

Muestras de (si proede): Z. TRANSICIONAL./ V. ST:MINALRS

4. Obstrvaciones y complicaciones: 
En primer lugar se analizaron las variables clínicas, estudiando la frecuencia de su distribución y sus porcentajes, tanto válido como acumulativo. Las variables cuantitativas se expresaron como media \pm desviación estandar (DE).

Las variables cualitativas se expresaron como frecuencias y porcentajes.

Para el análisis de los datos, aplicamos las siguientes pruebas estadísticas:

1. Prueba de Kolmogorov-Smirnov para estudiar si la distribución era de tipo normal.

2. Test de Student para comparar las medias de dos categorías dentro de una misma variable dependiente y el análisis de la varianza (razón F).

3. Tablas de contingencia con aplicación de $\mathrm{Chi}^{2}$ para establecer asociaciones significativas entre variables categóricas.

4. Análisis de regresión logística de Wald (de pasos hacia delante), para conocer las variables independientes que han influido en la tolerancia a la biopsia.

Se estableció significación estadística para valores de $\mathrm{p}$ menores de 0,05 con intervalo de confianza del $95 \%$.

\section{RESULTADOS}

La edad media en el momento de hacerse las biopsias fue de 67,2 $\pm 6,7$ años (50-80), siendo el volumen prostático medio de $47,7 \pm 24,18 \mathrm{cc}$ (16170).

Respecto al número de cilindros tomados (Tabla II y Figura 1), 43 pacientes fueron sometidos

TABLA II. NÚMERO DE CILINDROS TOMADOS.

\begin{tabular}{|l|l|l|l|}
\hline № de Cilindros & Frecuencia & Porcentaje & Porcentaje \\
\hline sextantes & 43 & 29,1 & acumulado \\
\hline $7-9$ & 38 & 25,7 & 29,3 \\
\hline$\geq 10$ & 67 & 45,3 & 55,1 \\
\hline Total & 148 & 100,0 & 100,0 \\
\hline
\end{tabular}

a biopsias sextantes, en 38 se realizaron de 7 a 9 tomas y en el resto (67) se recogieron entre 10 y 12 muestras. La mediana del número de cilindros recogidos por paciente fue de 8 cilindros (6-12).

De estos 148 pacientes, 50 habían sido sometidos previamente a 1 o más biopsias.

En cuanto a los antecedentes, cabe destacar que 21 pacientes tomaban antiagregantes y 9 anticoagulantes orales, todos ellos los habían suspendido entre 5 y 10 días antes de la prueba. Respecto a la patología rectal presente en estos pacientes, 29 pacientes presentaban hemorroides, de los cuales en 20 eran externas, en 6 internas y 3 casos presentaban hemorroides tanto externas como internas. Cinco pacientes habían presentado fisuras anales y 2 presentaban cirugías anales previas (Tabla III), no encontrándose asociación significativa entre el dolor experimentado en la prueba con la existencia de patología rectal o con cirugía a este nivel.

\section{Complicaciones tras la prueba:}

Hubo cierto sangrado en 81 casos pero sólo en uno fue importante, requiriendo de la colocación de una sonda vesical, si bien no requirió ingreso. En 1 caso se produjo un cuadro séptico tras la biopsia que precisó de ingreso durante 5 días. En 4 pacientes se produjeron reacciones vagales durante 0 tras la prueba que se resolvieron con maniobras posturales, y en otros 4 el dolor obligó a interrumpir el procedimiento.

\section{Tolerancia a la prueba:}

Se recogieron los datos de tolerancia en 147 casos, existiendo dolor intenso o insoportable globalmente en 25 casos $(16,9 \%)$ y nada

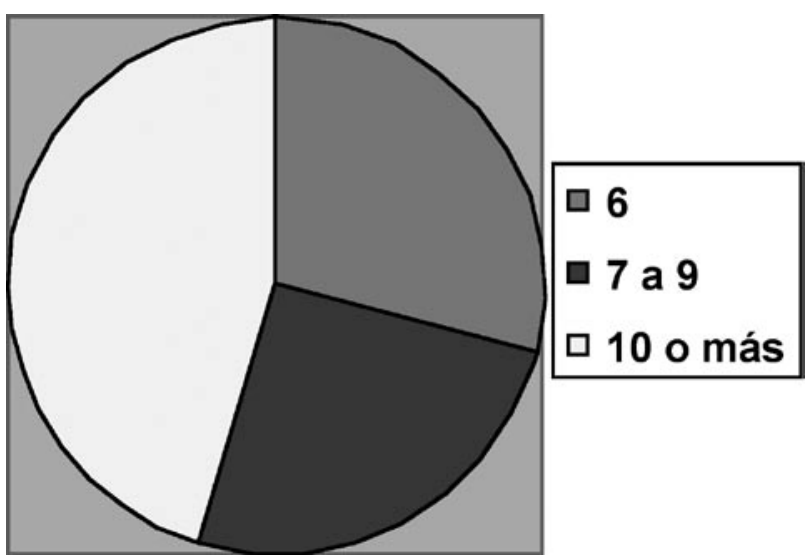

FIGURA 1. Porcentaje de pacientes en relación al número de cilindros tomados. 
TABLA III. PATOLOGÍA ANAL PRESENTE EN LOS PACIENTES SOMETIDOS A BIOPSIAS PROSTÁTICAS.

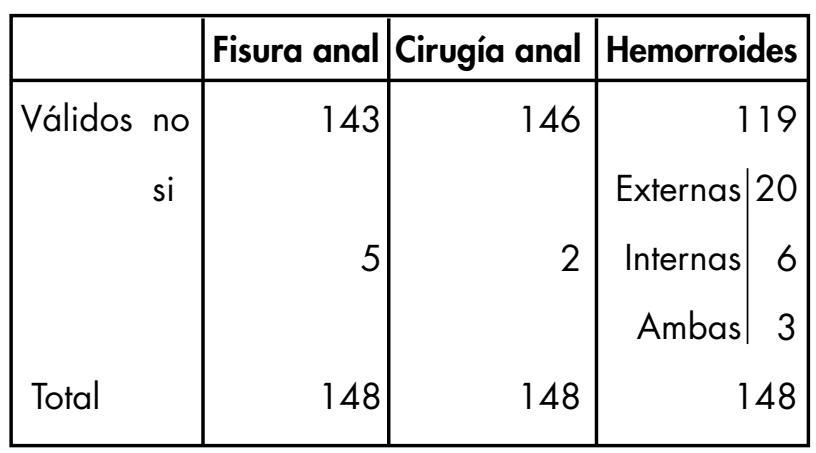

molesto en 45 pacientes $(30,4 \%)$. Se evidenció una asociación significativa entre el resultado del cuestionario del paciente y la percepción del médico que realizó la prueba $(p=0,0001)$ (Tabla IV y Figura 2).

La información previa sobre el procedimiento fue adecuada en la mayoría de los casos ( $n=124$, $83,7 \%$ ) según los propios pacientes, estando muy ansioso ante la prueba el $18,2 \%(n=27)$. El $29,1 \%$ $(n=46)$ de pacientes pensaba que se trataba de una prueba dolorosa antes de su realización. Sólo en 10 casos el tacto rectal fue doloroso, en 13 el paso del transductor y en 15 el movimiento del mismo en el recto (Tabla IV y Figura 2).

No obstante, en el $24,3 \%(n=36)$ de los casos la toma de cilindros fue dolorosa y en 77 pacientes $(52 \%)$ resultó molesta, siendo esta variable la que más influyó en la percepción global, demostrándose además que era la única variable independiente en el estudio de regresión logística (Wald) realizado (Tabla IV y Figura 2), de forma que la toma de biopsias es lo que más molesta por encima de otras maniobras (tacto rectal, introducción del transductor o movimiento del mismo) durante la realización de la prueba (dentro de esto, el ápex y el número de cilindros obtenidos es lo más importante). Sólo 14 pacientes no realizarían nuevamente la misma prueba o requerirían anestesia y el $89,9 \%$ (133) volverían a realizarla en las mismas condiciones.

En el $60 \%$ de los pacientes las biopsias de ambos lóbulos fueron igualmente dolorosos, en el $7,4 \%(n=11)$ el primer lóbulo biopsiado resultó más doloroso, mientras que en el 31,1\% fue peor el segundo lóbulo biopsiado. Prácticamente todos los cilindros que resultaron dolorosos o insoportables en la toma de muestras fueron los recogidos de la zona del ápex.

Se encontraron diferencias estadísticas $(p=0,005)$ en la prueba de $\mathrm{Chi}^{2}$ al estudiar una posible asociación entre el nivel de dolor y el número de pinchazos realizado, agrupados en 3 grupos, (sextantes, 7-9, $\geq 10$ ) (Tabla V), de manera que un mayor número de pinchazos se asoció significativamente con un mayor grado de dolor, así, el $37,2 \%$ de los pacientes sometidos a la toma de 6 cilindros consideraron la prueba como nada molesta, frente al $84,8 \%$ de los pacientes sometidos a la toma de 10 a más cilindros, quienes consideraron la prueba como molesta o dolorosa.

TABLA IV. TOLERANCIA A LA PRUEBA EN SUS DISTINTAS FASES.

\begin{tabular}{|c|c|c|c|c|c|c|}
\hline \multicolumn{2}{|c|}{ Tolerancia a la prueba } & \multirow{2}{*}{$\begin{aligned} \text { Global } \\
\\
45\end{aligned}$} & \multirow{2}{*}{$\begin{array}{r}\text { Tacto rectal } \\
75\end{array}$} & \multirow{2}{*}{$\begin{array}{r}\begin{array}{r}\text { Introducción } \\
\text { de la sonda }\end{array} \\
70\end{array}$} & \multirow{2}{*}{$\begin{array}{r}\begin{array}{l}\text { Movimiento } \\
\text { de la sonda }\end{array} \\
70\end{array}$} & \multirow{2}{*}{$\begin{array}{r}\begin{array}{r}\text { Toma de } \\
\text { cilindros }\end{array} \\
34\end{array}$} \\
\hline Válidos & Nada molesto & & & & & \\
\hline & Molesto & 77 & 62 & 64 & 61 & 77 \\
\hline & Doloroso & 23 & 9 & 12 & 12 & 26 \\
\hline & Insoportable & 2 & 1 & 1 & 4 & 10 \\
\hline & Total & 147 & 147 & 147 & 147 & 147 \\
\hline Perdidos & & 1 & 1 & 1 & 1 & 1 \\
\hline Total & & 148 & 148 & 148 & 148 & 148 \\
\hline
\end{tabular}




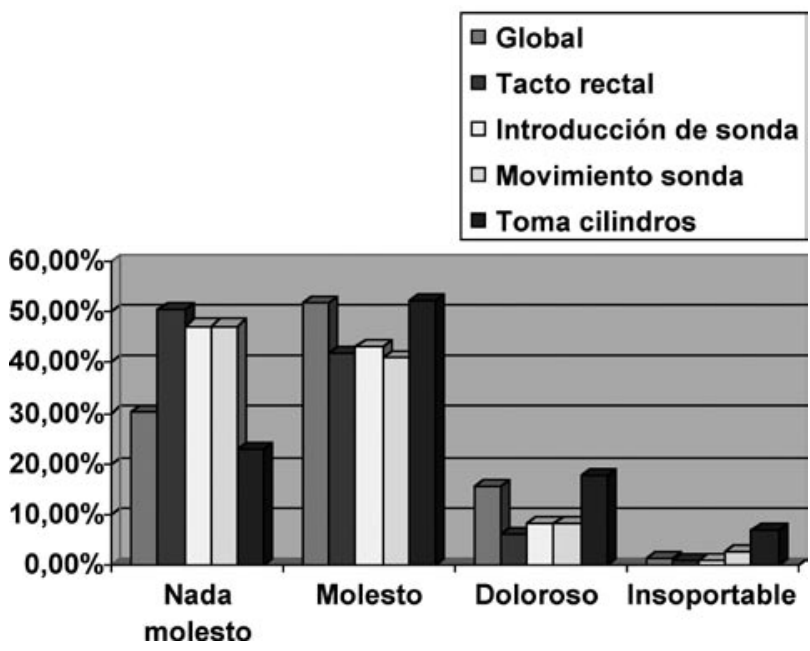

FIGURA 2. Grado de tolerancia a las distintas fases de la prueba.

No se encontró una asociación significativa entre la existencia de dolor derivado de los pinchazos con el nivel cultural del paciente, ni con el médico que realizó la biopsia.

\section{DISCUSIÓN}

Para la mayoría de urólogos, las molestias ocasionadas durante la realización de una biopsia prostática ecodirigida son mínimas e insignificantes por lo que no precisarían de anestesia, sin embargo, varios estudios como el de Collins y cols. (8) demostraron que hasta el $90 \%$ de pacientes consideraban esta prueba como dolorosa. En nuestro estudio, 102 pacientes $(69,4 \%)$, consideraron la prueba como al menos molesta, mientras que a $23(15,6 \%)$ les resultó dolorosa y $2(1,3 \%)$ la consideraron insoportable.
La administración del gel intrarectal de lidocaina con el fin de disminuir las molestias durante la prueba, fue evaluada previamente en otro estudio llevado a cabo por Chang y cols. (9) donde 108 pacientes fueron randomizados a recibir o bien un gel con lidocaina intrarectal o un gel sólo con lubricante. El único resultado significativo obtenido en ese estudio, fue que los hombres jóvenes experimentaban mayores molestias durante la prueba, pero no encontraron mejoría significativa en el grupo en los que se utilizaba el gel con lidocaina. En nuestro estudio, evidenciamos que el $89,9 \%$ de los pacientes realizarían las pruebas en la mismas condiciones, si bien encontramos una asociación significativa $(p=0,007)$ entre el número de cilindros y el dolor durante la obtención de los mismos, siendo mayor de lo esperado el dolor cuando se realizaron biopsias por encima de las sextantes. Teniendo en cuenta que en las guías europeas del cáncer de próstata de la Asociación Europea de Urología hay una mayor tendencia a la obtención de un mayor número de cilindros (10), la necesidad de algún tipo de anestesia adicional, parece evidente.

El bloqueo del plexo nervioso periprostático, es indudablemente un buen método para disminuir el dolor durante esta prueba, sin embargo en una revisión realizada por Davis y cols. (11) se evidenció que sólo el 11 \% de urólogos de Florida (EEUU) conocían esta técnica y hasta el $50 \%$ de estos no utilizaban ningún tipo de anestesia durante la realización de esta prueba.

En nuestra experiencia la biopsia múltiple ecodirigida de próstata es generalmente bien tolerada empleando únicamente un gel anestésico intrarrectal. No obstante, el número de punciones durante la biopsia ha sido el factor asociado al dolor de la prueba y al incrementarse aquel debería plantearse el empleo de algún tipo adicional de anestesia.

TABLA V. GRADO DE DOLOR EN FUNCIÓN DEL NÚMERO DE CILINDROS TOMADOS EN LA PRUEBA.

\begin{tabular}{|c|c|c|c|c|}
\hline Grado de dolor & sextantes & $7-9$ & $\geq 10$ & Total \\
\hline Nada molesto & $16(37,2 \%)$ & $8(21,1 \%)$ & $10(15,1 \%)$ & 34 \\
\hline Molesto & $23(53,4 \%)$ & $15(39,5 \%)$ & $39(59,1 \%)$ & 77 \\
\hline $\begin{array}{l}\text { Doloroso e } \\
\text { Insoportable }\end{array}$ & $4 \quad(9,3 \%)$ & $15(39,5 \%)$ & $17(25,7 \%)$ & 36 \\
\hline Total & 43 & 38 & 66 & 147 \\
\hline
\end{tabular}




\section{BIBLIOGRAFÍA Y LECTURAS RECOMENDADAS (*lectura de interés $y^{* *}$ lectura fundamental)}

*1. ISSA, M.M.; BUX, S.; CHUN, T.: "A randomized prospective trial of intrarectal lidocaine for pain control during transrectal prostate biopsy: the Emory University experience". J. Urol., 164: 397, 2000.

**2. DJAVAN, B.; ZLOTTER, A.R.; KRATZIK, C.: "PSA, PSA density, PSA density of transition zone, free/total PSA ratio and PSA velocity for early detection of prostate cancer in men with serum PSA 2,5 to 4,0 ng/ml”. Urology, 54: 517, 1999.

**3. REMZI, M.; FONG, Y.K.: "The Vienna-nomogram: validation of a novel biopsy strategy defining the optimal number of cores based on prostatic specific antigen, patient age and total prostate volume". J. Urol. 174: 1.256, 2005.

*4. DJAVAN, B.; RAMZI, H.: "Repeat prostate biopsy: who, how and when? A review". Eur. Urol., 42: 93, 2002.

*5. DE SIO, M.; D`ARMIENT0, M.; DI LORENZO, G. y cols.: "The need to reduce patient discomfort during transrectal ultrasonography-guided prostate biopsy: what do we know?". BJU Int., 96: 977, 2005.

6. MATLAGA, B.R.; ESKEW, A.; McCULlOUGH, D.L.: "Prostate biopsy: indications and technique". J. Urol., 169: 12, 2003.

*7. AUTORINO, R.; DE SIO, M.; DI LORENZO, G. y cols.: "How to decrease pain during transrectal ultrasound guided prostate biopsy: a look at the literature". J. Urol., 174: 2091, 2005.

8. COLLINS, G.N.; LLOYD, S.N.; HEHIR, M. y cols.: "Multiple transrectal ultrasound-guided prostate biopsies true morbidity and patient acceptance". Br. J. Urol., 71: 460, 1993.

9. CHANG, S.S.; ALBERTS, G.; WELLS, N. y cols.: "Intrarectal lidocaine during transrectal prostate biopsy: results of a prospective double-blind randomized trial". J. Urol., 166: 2178, 2001.

10. AUS, G.; ABBOU, C.C.; BOLLA, M. y cols.: "EAU guidelines on prostate cancer". EAU., March: 13, 2005.

**11. DAVIS, M.; SOPHER, M.; KIM, S.S. y cols.: "The procedure of transrectal ultrasound guided biopsy of the prostate: a survey of patient preparation and biopsy technique". J. Urol., 16: 566, 2002. 